\title{
Antioxidantes: perspectiva actual para la salud humana
}

\author{
Antioxidants: present perspective \\ for the human health
}

\begin{abstract}
The aim of this paper is to highlight data related to health and antioxidants. According to the literature, these substances may participate to neutralize the excess of free radicals in oxidation mechanisms. The production of free radicals, a usual biological phenomenon, is regulated by different metabolic routes, since they are the first line of defense in the human body. Even though they are important in order to maintain a healthy body, the imbalance between endogenous antioxidants and free radicals, known as oxidative stress, is associated with different diseases and human aging. This paper also presents some sources of exogenous antioxidants, focusing on fruits and other vegetables and presents data from a local exploratory study about antioxidants consumption. Key words: antioxidants, free radicals, oxidative stress, human health.
\end{abstract}

Marta Coronado H. Salvador Vega y León Rey Gutiérrez T. Marcela Vázquez F. Claudia Radilla $V$.

Universidad Autónoma Metropolitana
Unidad Xochimilco, México.
Dirigir la correspondencia a:
Profesora
Marta Coronado H.
Universidad Autónoma Metropolitana. Unidad Xochimilco, México.
E-mail: mcoronado@correo.xoc.uam.mx

Este trabajo fue recibido el 14 de Enero de 2014 , aceptado con modificaciones el 21 de Enero de 2015 y aceptado para ser publicado el 25 de Febrero de 2015.

\section{INTRODUCCIÓN}

El objetivo de este trabajo es contribuir con un aporte que enriquezca el conocimiento actual sobre los antioxidantes y su papel en la salud humana. En la actualidad causa impacto entre los consumidores el denominado "mercado de la salud" que cada día se expande en el mundo (1); este incluye los alimentos con antioxidantes considerados alimentos funcionales y que entre otras definiciones, se pueden describir como productos alimenticios (animal o vegetal) naturales o industrializados que forman parte de la dieta diaria (2) y que además de aportar nutrientes, tienen otros componentes bioactivos. Pueden contener ingredientes adicionales tales como fibra, vitaminas, minerales o eliminar algún compuesto (grasas saturadas o azúcares) con el fin de hacerlos más nutritivos.

El trabajo incluye un estudio exploratorio en la ciudad de México, el cual establece además del consumo, la percepción de los participantes sobre los productos con antioxidantes que tienen amplia difusión actual.

\section{DEFINICIÓN DE ANTIOXIDANTE}

Un antioxidante dietético es una sustancia que forma parte de los alimentos de consumo cotidiano y que puede prevenir los efectos adversos de especies reactivas sobre las funciones fisiológicas normales de los humanos (3).

Las propiedades antioxidantes no sólo deben estudiarse por sus interacciones químico-biológicos, sino por su función en el deterioro oxidativo que afecta a los alimentos (4). Se utilizan en la industria alimentaria adicionados a las grasas u otros productos para retrasar los procesos de oxidación, en tanto previenen el comienzo de la rancidez oxidativa (grasas) (5).

\section{PROCESO DE OXIDACIÓN Y LOS AGENTES PROMOTORES}

Asociado a la función antioxidante se considera el proceso de óxido-reducción que remite a dos momentos básicos: a) oxidación que implica pérdida de electrones de hidrógeno con la ganancia de oxígeno en la molécula, b) reducción que significa ganancia de electrones de hidrógeno con la pérdida de oxígeno. Así el oxidante se reduce al reaccionar con aquella molécula que oxida. Este proceso es cotidiano en el organismo humano y representa el conocido par óxido-reductor o balance redóx (6).

Es pertinente aclarar que a veces el término oxidación sólo se remite a ciertas sustancias aisladas (oxidación de grasa), pero no hay una comprensión específica de que esta denominación (oxidación) se refiere a procesos celulares, internos que conllevan la aparición de enfermedades. Así, no sólo hay que valorar los mecanismos de defensa del organismo, sino también las especies involucradas, tales como los radicales libres que se analizan en el apartado siguiente.

\section{RADICALES LIBRES, PRODUCCIÓN Y ACCIÓN}

Un radical libre es aquella figura química que tiene en su estructura uno o más electrones no apareados. Es altamente reactiva y clave para formar otros radicales libres en cadena, (7), además por la vida media que es de microsegundos, ocurre 
una rápida propagación con moléculas aledañas y mayor daño potencial. De hecho un radical libre puede afectar 1 millón de moléculas durante la reacción en cadena (8). Los compuestos en cuestión forman parte de las llamados especies reactivas del oxígeno (ERO) o ROS (Reactive Oxigen Species) (7).

Los radicales libres se liberan durante el metabolismo humano, y también se producen por contaminantes ambientales, (atmosféricos, acuáticos, de suelos), radiaciones (ultravioleta, gamma, hertziana), entre otros. Se pueden relacionar con el consumo o uso de tóxicos como el alcohol, tabaco y drogas o debido a una alimentación no adecuada, exposición a fertilizantes o pesticidas. Se incluye además el metabolismo de algunos químicos y elevado estrés físico o psíquico (5).

La figura 1 explica la interacción entre radical libre y antioxidante.

\section{ESTRÉS OXIDATIVO}

No hay que confundir este mecanismo celular con el estrés cotidiano derivado de las problemáticas que enfrenta el organismo humano en su vida diaria (trabajo, familia, trámites, entre otras). El estrés oxidativo es un término asociado a las células y a la acción de un radical libre que le afecta, (9) así en condiciones normales se da un equilibrio entre la producción de radicales libres u otras especies reactivas con los mecanismos antioxidantes (exógeno y endógeno). Este equilibrio permite que la toxicidad por oxidación sea menor y con menos daño celular. Cuando se rompe el equilibrio, éste se podrá asociar con un déficit en el sistema antioxidante o por la proliferación descontrolada de los radicales libres (6).

\section{CONTROL BIOLÓGICO DE LOS \\ PROCESOS DE ÓXIDO-REDUCCIÓN}

Existen dos vías fundamentales para proteger al organismo de los radicales libres; enzimáticos y no enzimáticos (endógenos y exógenos) y su interacción puede ser intracelular o extracelular (8). La vía endógena requiere apoyo externo y por ello se recomiendan los antioxidantes exógenos cuyo papel es relevante dado que son los que pueden formar parte de la dieta diaria, como los indicados en un apartado posterior.

A continuación se analiza un fenómeno biológico natural asociado con la salud humana y con los antioxidantes.

\section{PERSPECTIVA DE LA RELACIÓN \\ ANTIOXIDANTES-ENVEJECIMIENTO HUMANO}

Las teorías del envejecimiento señalan por una parte, la programación genética con una respuesta predeterminada de cada organismo y por otra un proceso no genético que incluye a los radicales libres o el estrés oxidativo (10).

Desde 1956 Denham Harman de la Universidad de Nebraska, planteó la relación entre radicales libres y envejecimiento. Se señaló que la expectativa de vida humana podría aumentar al disminuir los efectos del proceso oxidativo. Así las especies reactivas del oxígeno, entre otros, los radicales libres, pueden alterar la membrana interna o el ADN mitocondrial lo que conlleva más producción de ERO, en consecuencia más daño y aumento del estrés oxidativo, al producirse más oxidantes y perderse el equilibrio requerido por la célula. Se señala (10) que el genoma mitocondrial es susceptible al ataque de los radicales libres que la misma mitocondria produce. En este
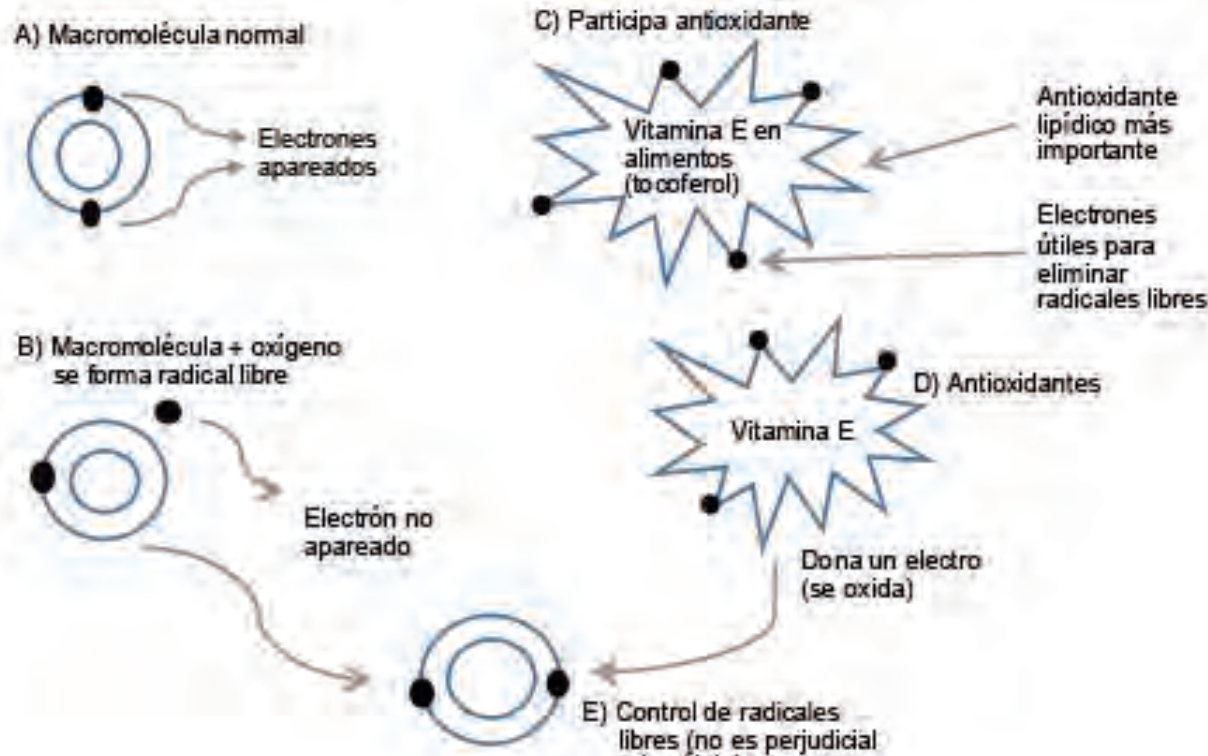

E) Control de radicales libres (no es perjudicial a la célula) 
marco la participación mitocondrial, y la presencia de estrés oxidativo podrían asociarse con la patogenia que conduce a la destrucción celular propia del envejecimiento

Durante el envejecimiento también disminuye la protección antioxidante y puede haber más ataque a las moléculas blanco. Sin embargo, algunas moléculas como carbohidratos, lípidos o proteínas pueden tener un efecto particular.

En el caso de los lípidos, durante la peroxidación se produce malondialdehido, el cual reacciona con lípidos y proteínas formando las denominadas bases de Schiff conjugadas, que finalmente enlazan al producto fluorescente insoluble que se acumula en los tejidos (lipofucsina) y que se toma como dato de vejez (11).

Por otra parte, la literatura (11) señala que la longevidad parece aumentar en concordancia con los niveles de antioxidantes en la dieta y con una reducción calórica; lo que puede propiciar una menor degradación de las mitocondrias, del metabolismo celular y del consumo de oxígeno. También una situación de constante estrés oxidativo durante la vejez puede alterar el sistema inmune. Se ha observado una disminución de antioxidantes (como el glutatión) durante el proceso normal de envejecimiento, sobretodo en la sangre y algunos órganos, tanto en animales como en humanos. Estos cambios degenerativos del sistema inmune pueden conducir a la formación de cataratas, inicios de Alzheimer, Parkinson o problemas cardiovasculares. Por lo anterior un buen sistema inmune se asocia con salud y longevidad (12).

Para fundamentar la relación vejez-antioxidante Cesari, et al (2004) realizaron un estudio (13) en personas de la tercera edad (>75.3 años) con el análisis de la fuerza muscular y el rendimiento físico (fuerza de extensión de la rodilla, velocidad al caminar, equilibrio y capacidad para levantarse).

Se evaluó la ingesta diaria de antioxidantes y la concentración plasmática de éstos con los parámetros de rendimiento físico señalados antes. Se midió la ingesta de alimentos con particular atención al consumo de vitamina $C$, vitamina $E$, $\beta$-caroteno y retinol. Entre otros se observó una correlación significativa entre la ingesta diaria de vitamina $C$ y $\beta$-caroteno y la fuerza de extensión de rodilla en los sujetos participantes (13).

Es importante mencionar que la vejez no afecta por igual a todos los individuos y a veces la edad cronológica y la biológica no coinciden, de ahí la necesidad de estudios longitudinales con diversas poblaciones.

\section{RELACIÓN ANTIOXIDANTES-EJERCICIO FÍSICO Y SALUD HUMANA}

Se reconoce que la práctica frecuente y sistemática de ejercicio físico es recomendable para la salud y el aumento de las defensas antioxidantes, entre otros efectos positivos. Sin embargo, no escapa al análisis que durante el ejercicio aumenta la producción de radicales libres que afectan el tejido muscular, el hígado, la sangre u otros (14).

Es posible que el ejercicio físico aislado aumente el daño oxidativo y en contraposición el entrenamiento regular y sistemático reduzca estos efectos, pero sin someter al organismo al ejercicio excesivo y al sobreentrenamiento que conduce a un estado de estrés oxidativo. Algunos estudios (15) indican que al incluir en la dieta de atletas entrenados alimentos con antioxidantes, redujo el estrés oxidativo. Además la producción de ácido láctico durante el ejercicio puede convertir el superóxido (radical poco dañino) a un hidroxilo (altamente lesivo para la célula) (15).

Frente a este marco, cabe preguntarse si el sistema de defensa de antioxidantes endógenos que tiene el organismo puede enfrentar las especies reactivas del oxígeno de manera eficiente o si, como apoyo, siempre deben recomendarse antioxidantes dietarios durante el entrenamiento físico. Al respecto se sugieren más estudios longitudinales con marcadores distintos y diferentes periodos de actividad física.

\section{OTROS APORTES SOBRE LA RELACIÓN ANTIOXIDANTE-SALUD}

De acuerdo con Núñez (2011) se han estudiado alrededor de 100 enfermedades y su relación con el desbalance del sistema oxidativo, entre otras: cardiovasculares, cáncer, gástricas, respiratorias, neurológicas y del sistema endocrino. Entre éstas las de tipo cardiovascular tienen amplia evidencia. La oxidación de las LDL (lipoproteínas de baja densidad) colesterol parece representar la "Ilave maestra" en el desarrollo de la ateroesclerosis, en tanto pueden ser citotóxicas de las células endoteliales y bajar la motilidad del tejido macrofágico. Se plantea que la vitamina $E$ que es transportada por las LDL colesterol puede reducir los procesos de oxidación (16). Otros antioxidantes con acción de vasodilatadores y vasoprotectores, además de antitrombóticos, antilipémicos o antiescleróticos (17), son los polifenoles cuya fuente y función se analizará más adelante.

En el caso de la diabetes los posibles mecanismos de los antioxidantes se relacionan con la inhibición en el intestino de la digestión de los carbohidratos, en particular la glucosa, de la cual también se modula su liberación por el hígado. Podrían también estimular la secreción de insulina en el páncreas y activar los receptores de la misma y de alguna manera activar la recaptura de glucosa en los tejidos blanco para la hormona. Otros efectos pueden ser la modulación de las rutas genéticas (9). Un dato relevante es que la metformina que se utiliza para el tratamiento de pacientes diabéticos resulta un poderoso antioxidante que disminuye la formación de radicales libres (18).

Respecto al cáncer se señala (5) que si los radicales libres afectan el DNA (ácido desoxirribonucleico) pueden ocurrir mutaciones que en su momento se transforman en células cancerosas. Se señala (8) la relación entre cáncer gástrico derivado de la presencia de Helicobacter pilori, bacteria que causa grastritis crónica y que puede conducir a lesiones precancerosas relacionadas con el estrés oxidativo.

En el caso del cáncer de mama aumenta la evidencia de que el riesgo de esta enfermedad, asociada con los genotipos humanos relacionados al estrés oxidativo, pueden modificarse con el consumo de frutas y vegetales. Se estudia la variación genética (19) de diversas enzimas que participan en la protección endógena del proceso de óxido-reducción del organismo y su respuesta hacia el consumo exógeno de antioxidantes provenientes de frutas y vegetales.

Es posible que las enfermedades neurodegenerativas sean de las más estudiadas en el contexto del estrés oxidativo. Se ha advertido el aumento del deterioro de proteínas específicas por la presencia elevada de las especies reactivas del oxígeno. De igual manera en algunas zonas del cerebro ocurre la disminución de algunos metales de transición $(\mathrm{Fe}+2 \rightarrow \mathrm{Fe}+3)$ propias del efecto oxidativo, lo cual agrava la enfermedad. El metabolismo de glutatión, también se ve afectado lo cual se asocia con enfermedades como Parkinson, Alzheimer u otras degenerativas del sistema neurológico (7). También la literatura (17) aborda el efecto benéfico de los antioxidantes (polifenoles) en el daño hepático y gástrico producido por ingesta de alcohol y la protección del intestino o como coadyuvante en el tratamiento de cataratas propias de la diabetes, entre otras.

De igual manera (20) se ha estudiado la relación de los antioxidantes con la artritis reumatoide, anemia, síndrome 
metabólico, esclerosis múltiple, trastornos nefrológicos, pancreatitis, arrugas prematuras, resequedad de la piel, dermatitis y asma entre otros padecimientos que en la actualidad abordan las investigaciones en curso.

Como en toda temática de salud, el diagnóstico, tratamiento o prevención requiere de años de estudio y de herramientas diversas para el análisis, sobre todo, en la actualidad que cada día surgen nuevas tecnologías para el trabajo experimental. Se requieren bases sólidas para apoyar los estudios de este campo científico y su traslado a la población en general.

\section{FUENTES EXÓGENOS DE ANTIOXIDANTES}

Entre los antioxidantes hay varias familias de principios activos como los polifenoles y los fitoestrógenos. Entre los primeros se encuentran los flavonoides (figura 2) y los taninos, ampliamente estudiados.

Entre los flavonoides (21) se pueden señalar sólo como ejemplo las antocianidinas (rojo-azulado de las fresas), catequinas (té verde y negro), citroflavonoides (naranjina, que da sabor amargo a lo naranja, limón, toronja), isoflavonoides (genisteína y daidzaína presentes en soya y sus derivados). Protoantocianidinas en semillas de uva y vino tinto.

Las dosis normales de ingesta de flavonoides pueden ser de 20-26 mg/día contenidos en frutas y verduras (manzanas, naranjas, guayabas, uvas). Sin embargo, hay que alertar sobre el consumo excesivo que pueden representar fórmulas comerciales de antioxidantes y mezclas herbales que se producen en gramos y no miligramos (como lo recomendado) y conducirían a problemas de toxicidad (21).

Otro tipo de antioxidantes son los taninos (polifenoles) presentes en el vino, con su característica de astringencia. No sólo son útiles a la industria alimentaria sino también en la de cosméticos (9).

Respecto a los fitoestrógenos (isoflavonas lignanos, flavonoides) se encuentran particularmente en las proteínas de la soya o sus derivados. Su uso más importante se asocia con la terapia de reemplazo hormonal para mujeres con síntomas de menopausia y osteoporosis durante el climaterio (2). Los compuestos relevantes son la genisteína y daidzeína que también se biosintentizan en abundancia en la cascarilla de semillas de linaza y centeno.

Entre los productos con antioxidantes, más consumidos, están aquellos con: vitamina E: aguacate, aceite de oliva, arroz integral, frutas secas; con vitamina C: acelgas, tomates (licopeno), todos los cítricos (limón, naranja, mandarina), además kiwi, fresa, guayaba; con $\beta$-caroteno: zanahoria, espinacas, mango, melón; con flavonoides: té verde, vino, manzana, o peras. Además hay antioxidantes en el ajo, cebolla, ginseng, ginko, avena, sauco (gripa), hierbabuena, menta, albaca, jamaica, chaya (Yucatán), calabacita, betabel y verdolagas (9). El tomate de árbol tiene una cantidad moderada de antioxidantes y el noni una cantidad elevada (22).

Se han estudiado ampliamente frutos rojos como el arándano (23) de diferentes familias botánicas y se ha evaluado el contenido de antioxidantes (proantocinidinas) por ración del arándano americano: compota de arándano entero: 24 $\mathrm{mg} / 70 \mathrm{~g}$; seco edulcorado: $32 \mathrm{mg} / 40 \mathrm{~g}$; coctel de zumo: 30 $\mathrm{mg} / 240 \mathrm{mg}$. Estos contenidos de antioxidantes es posible mejorarlos en algunos frutos rojos (fresa) con la selección de nuevos genotipos que ofrezcan mayor eficiencia. Los estudios indican (24) que la actividad antioxidante se ve afectada por el tipo de fruto, la especie y la variedad y si los frutos rojos (fresa) tienen de 2 a 11 veces más antioxidantes (poliofenoles, flavonoides y antocianinas) que la manzana o el kiwi; el mejorar las variedades es una tarea actual.

Un dato dietético a recordar es que las moléculas fenólicas tienen amplia afinidad por las proteínas (25) por lo que su capacidad antioxidante puede disminuirse in vivo, y se alerta sobre la ingestión de los frutos rojos (fresa, arándano) en combinación con leche, lo cual podría alterar la efectividad antioxidante de los mismos. Otro aspecto es la biodisponibilidad, que implica la cantidad de nutrimentos que se digieren, absorben y se metabolizan, por ello el contenido total de antioxidantes de un alimento es importante, pero más su biodisponibilidad, en tanto no los más abundantes son los más activos en el organismo, sea por absorción intestinal baja, por un mayor proceso metabólico, o excreción rápida (17).

Un alimento con antioxidante de alto consumo es la guayaba (Psidium guajava L.) que contiene vitaminas $A, C$ y polifenoles. Al estudiarse la fruta fresca, la piel, la pulpa y toda la capa externa (casco) se observa un mayor contenido de polifenoles (base húmeda) en la piel del fruto y después en la pulpa y el casco (26). De hecho, la piel de las frutas tiene mayor capacidad antioxidante que la pulpa, además la cocción reduce esta actividad y baja la concentración de polifenoles (27).

Otra fuente de antioxidantes (procianidinas, antocianinas

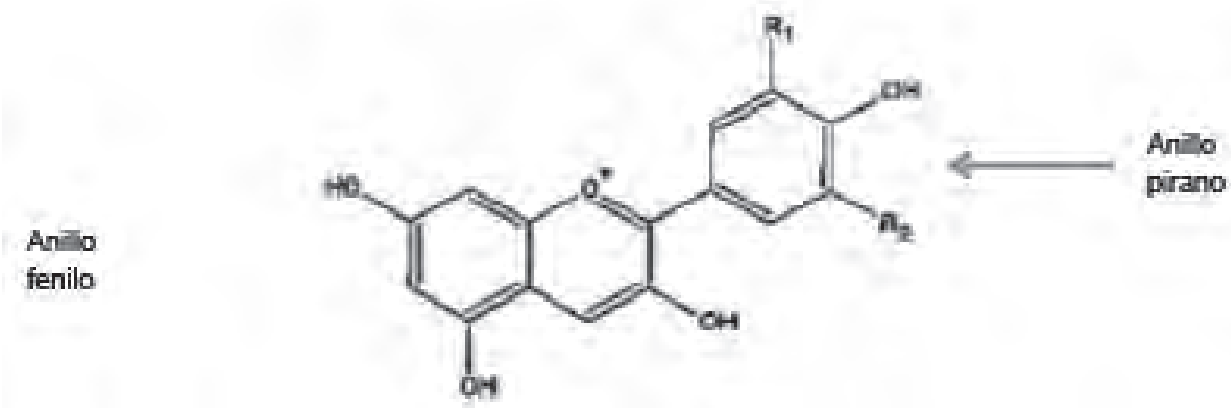

Fuente: Modificado de Drago, López Sainz, 2006. 
y flavononas) es el cacao y sus productos (licor de cacao, chocolate amargo, cocoa) ricos en catequinas, (15-20\% p/p) (28).

Perea, Cadena y Herrera, (2009) observaron que el chocolate amargo tiene alto contenido de polifenoles en tanto que el chocolate de mesa con azúcar, clavo y canela tiene un aporte intermedio y la cocoa con grasa vegetal tiene un contenido menor. Este último tiene valores superiores al de algunas frutas. Se podría considerar que comparado con otros alimentos los polifenoles de cacao y sus derivados se sitúan en este orden: vino tinto $\rightarrow$ cacao $\rightarrow$ té verde $\rightarrow$ manzana $\rightarrow$ pera $\rightarrow$ kiwi.

En México se ha estudiado la actividad antioxidante de la tuna no sólo para producir alimentos funcionales sino para la cosmetología o productos farmacéuticos (29). Este fruto contiene compuestos bioactivos como vitamina $C$, vitamina $E$ y polifenoles. Los aspectos físicos, químicos y biológicos (especie, estado de madurez, precosecha y poscosecha) influyen en la cantidad de nutrientes presentes. La tuna púrpura tiene menor capacidad antioxidante que las verdes o amarillas, y menor en las blancas.

Se han analizado diferentes variedades de maíz mexicano con antioxidantes los que son parte de la alimentación local (maíz pigmentado). Los estudios (30) indican que el contenido de compuestos fenólicos varía de acuerdo con los diferentes tipos de maíz, aunque éstos se observen con la misma pigmentación.

En México el tomate (Solanum lycopersicum) es de consumo diario, rico en potasio, ácido fólico, B-caroteno, vitamina $C$, Vitamina e, flavonoides y licopeno por lo que presenta una buena fuente de antioxidantes para enfrentar el estrés oxidativo. Incluso una dieta que incluya alimentos procesados de tomate (alto en licopeno) como salsas, jugos o pasta pueden disminuir la peroxidación lipídica y la oxidación de LDL colesterol (31). Un dato importante señala que el jugo de tomate almacenado por un año puede mantener su composición antioxidante debido a la estabilidad del licopeno, compuestos fenólicos y flavonoides totales. Así el procesamiento no deteriora la actividad biológica y mejora la biodisponibilidad del licopeno.

Otros alimentos locales como el agua de jamaica, tortillas, frijol, elote, y la bebida pozol (Chiapas, México), también tienen efectos antioxidantes (32).

En plantas medicinales, se han realizado estudios sobre el contenido de polifenoles y la actividad antioxidante. Se puede señalar que la Passiflora (33) se asocia con propiedades farmacológicas (sedativas, antiespasmódicas o antibacteriales). En diversos países se usa en problemas de ansiedad, insomnio, epilepsia o espasmos. Su capacidad antioxidante es mayor en las hojas que en los frutos.

Finalmente cabe alertar sobre el consumo de frutas o verduras troceadas que se venden en diferentes puntos comerciales (cafeterías, supermercados, tianguis, puestos en la calle), porque el pelado y cortado incrementa la actividad metabólica del producto, incide en los compuestos fitoquímicos presentes y en consecuencia en la capacidad antioxidante. De hecho el oscurecimiento del tejido, producto de la degradación de fenoles (oxidación por la polifenoloxidasa) no sólo altera la apariencia del alimento también disminuye la actividad antioxidante (34).

\section{ESTUDIO LOCAL EXPLORATORIO SOBRE EL CONSUMO DE ALIMENTOS CON ANTIOXIDANTES EN LA CIUDAD DE MÉXICO}

Se realizó un estudio preliminar (encuesta con preguntas cerradas) con dos grupos de trabajo: 165 estudiantes univer- sitarios en su última etapa de formación profesional en el área biológica (nutrición, medicina y química de alimentos). Lo anterior aseguró una mayor información sobre la temática estudiada (antioxidantes y su consumo), el otro grupo fue integrado por 135 sujetos de población abierta, con poder adquisitivo medio, (padres de familia, profesores de educación básica, amas de casa y ancianos). Fue un estudio transversal, observacional y descriptivo.

Se observó lo siguiente: los estudiantes universitarios identificaron en porcentaje variable (en una batería de reactivos) los conocimientos básicos: a) concepto de radicales libres $(96.6 \%)$, b) concepto de estrés oxidativo $(59.3 \%)$, c) causas del estrés oxidativo $(60.6 \%), d)$ ventajas de antioxidantes para el tratamiento de enfermedades crónico degenerativas (75.3\%).

Respecto al envejecimiento los estudiantes consideraron (69.5\%) que evita la aparición temprana de edad avanzada. Este último dato refuerza la idea difundida en la publicidad comercial, sobre el consumo de antioxidantes y su relación con el envejecimiento humano.

De igual manera la identificación de una molécula de antioxidantes (antocianina) y otra de radical libre fueron bien reconocidas ( $98.6 \%$ y $100 \%)$. Entre los alimentos naturales con antioxidantes los valores fueron: guayaba (36.9\%), manzana (30.8\%), melón (15.7\%) y chile (16.4\%), lo que indica un buen acercamiento a los alimentos que se consideran entre los más señalados como fuente de antioxidantes (guayaba y manzana).

Entre los alimentos industrializados el de mayor porcentaje fue el jugo de arándano (36.7\%) y el té verde (30.0\%). Es posible que estos dos productos sean reconocidos por su amplia publicidad en los medios televisivos de México.

En cuanto a la importancia que un profesional de la salud incluya en su saber disciplinario la temática estudiada (antioxidantes y salud), (75.3\%) de los universitarios lo considera relevante.

En el otro grupo estudiado destaca lo siguiente: igual que entre los estudiantes universitarios los diferentes grupos sociales (64.0\%) señalaron el consumo de alimentos con antioxidantes como una forma de prevenir la aparición temprana de edad avanzada. Así mismo; 61.3\% consideró como benéfico el consumo de alimentos con antioxidante para el retardo del envejecimiento. Es posible que ambas respuestas estén asociadas con la difusión continua (sobre todo en televisión) de los efectos positivos de este tipo de alimentos para conservar una imagen de juventud.

Al identificar los alimentos con antioxidantes de mayor consumo, 50.4\% de los participantes indicó dos frutos: manzana (28.4\%) y guayaba (22.1\%). Los frutos rojos, ricos en antioxidantes también fueron señalados (84.4\%) como de consumo frecuente: fresas $(45.6 \%)$, ciruela negra $(24.0 \%)$ y arándano (14.8\%).

En cuanto a alimentos industrializados $84.7 \%$ seleccionó tres productos: jugo de manzana (33.5\%), té verde (29.3\%) y jugo de arándano (21.9\%). Se puede mencionar que los tres son ampliamente publicitados y ofertados en los puntos de venta, sobretodo en supermercados de la Ciudad de México.

La frecuencia de consumo semanal de alimentos con antioxidantes se distribuyó en 1 a 2 veces (45.3\%) y 3 a 4 veces (29.3\%) lo que representó al $74.6 \%$ de los participantes.

El lugar de compra fue esencialmente los supermercados (64.3\%) comparado con otros puntos de venta (tienda de barrio, tianguis o tiendas especializadas).

Las razones de consumo se asociaron con la información oral o leída sobre los beneficios para la salud (64.4\%) y con el gusto por estos productos (27.1\%). 
Finalmente los medios de comunicación que más utilizan los participantes (66.4\%) para informarse sobre alimentos con antioxidantes fueron: televisión (31.4\%), revistas de difusión o de entretenimiento (25.0\%). Otros medios como internet, radio o periódico fueron los de menor elección.

Este estudio preliminar demostró que tanto entre los estudiantes universitarios como en población abierta se perciben los beneficios del consumo de alimentos con antioxidantes y ya se incluyen en la dieta diaria.

Sin embargo, se advierte la necesidad de estudios de tipo longitudinal con mayor profundidad y de tal forma que no sea un evento de aceptación y consumo de alimentos por un factor más de moda, que de salud.

\section{CONCLUSIONES}

La literatura citada sugiere la necesidad de profundizar los estudios experimentales y epidemiológicos para caracterizar de mejor manera, no sólo el estrés oxidativo sino la relación de este fenómeno con la salud humana. De igual forma se requiere identificar más y mejores marcadores, incluso genéticos con los que se puedan estudiar diversos grupos poblacionales que permitan no sólo recomendar terapias antioxidantes, para el tratamiento, sino en primera instancia, para prevenir riesgos de padecimientos asociados con los radicales libres.

La investigación científica aporta datos sobre el beneficio del consumo cotidiano de alimentos con antioxidantes, sobre todo de frutas y verduras y la protección que pueden proporcionar para prevenir los riesgos de padecimientos, aunque se reitera que se requieren más estudios.

Por otra parte, al ampliarse la oferta de comunicación (internet, televisión, radio periódico, revistas) la difusión masiva de información en torno a la alimentación, ha permitido que la población aumente la tendencia de consumo de los alimentos con antioxidantes que además en México, tiene buena oferta en el mercado de productos naturales o industrializados. Así, la búsqueda actual de alimentos saludables ha resultado particularmente benéfica para la industria y para el consumidor. No obstante, cabe alertar a la población que el consumir alimentos con antioxidantes es una elección alimentaria que debe guiarse por convicción y conocimientos sobre sus efectos benéficos para la salud y no por moda alimentaria.

\section{RESUMEN}

El objetivo de este artículo es resaltar aspectos sobre los antioxidantes y la salud. De acuerdo con la literatura, los antioxidantes pueden neutralizar el exceso de radicales libres durante la actividad oxidativa, propia del organismo. La producción de radicales libres, un evento natural, es regulado por diferentes rutas metabólicas, porque representan la primera línea de defensa de los seres vivos. Sin embargo, aunque son relevantes para mantener la salud, el desbalance entre antioxidantes endógenos y radicales libres (estrés oxidativo) se asocia con diferentes enfermedades o con el envejecimiento humano, como se analiza en este texto. El artículo también incluye algunas fuentes de antioxidantes exógenos, sobre todo frutas y otros vegetales, así como datos de un estudio local exploratorio sobre consumo de antioxidantes.

Palabras clave: antioxidantes, radicales libres, estrés oxidativo, salud humana.

\section{BIBLIOGRAFÍA}

1. Vega, S. Innovaciones alimentarias al inicio del siglo XXI. El caso de los llamados alimentos y sustancias funcionales. En: Coronado, M., comp. Innovación tecnológica en el futuro de los profesionales en áreas biológicas. Un texto para estudiantes universitarios. México, D.F.: UAM-X. 2003; p. 223-59.

2. Drago, M., López, M., Saínz, T. Componentes bioactivos de alimentos funcionales de origen vegetal. Rev Mexicana Ciencias Farmacéuticas. 2006; 37 (4): 58-68.

3. Patthamakanokporn, O., Puwastien, P., Nitithamyong, A., Sirichakwal. P. Changes of antioxidant activity and total phenolic compounds during storage of selected fruits. J Food Composition Analysis. 2008; 21, 241-8.

4. Pastene, E. Estado actual de la búsqueda de plantas con actividad antioxidante. Boletín Latinoam Caribe Plantas Med Aromáticas. 2009; 8 (6), pp. 449- 55.

5. Llancari, A., Matos, A. Valoración de los nutrientes y antioxidantes en la salud humana e industria alimentaria. En: Universidad Peruana Unión. I Congreso Nacional de Investigación. Perú, Lima, 2-4 noviembre, 2011.

6. Quintanar, M., Calderón, J. La capacidad antioxidante total. Bases y Aplicaciones. Rev Educación Bioq. 2009; 28 (3):89-101.

7. Núñez, A. Terapia antioxidante, estrés oxidatívo y productos antioxidantes: retos y oportunidades. Rev Cubana Salud Pública. 2011; 37 (suppl.): 644-60.

8. Zamora, J. Antioxidantes: micronutrientes en lucha por la salud. Rev Chil Nutr. 2007; 34 (1): 17-26.

9. Reyes, A., Galicia, M., Carrillo, M. Antioxidantes: la magia de lo natural. Rev Tlatemoani. 2011; (8): 1-16.

10. Céspedes, E., Rodríguez, K., Llópiz, N., Cruz, N. Un acercamiento a la teoría de los radicales libres y el estrés oxidativo en el envejecimiento. Rev Cubana Invest Biomed. 2000; 19 (3), pp. 186-90.

11. Zorrilla, A. El envejecimiento y el estrés oxidativo. Rev. Cubana Invest. Biomed. 2002; 21 (3): 178-85.

12. De la Fuente, M. Effects of antioxidants on immune system ageing. European J Clin Nutr. 2002; 56 (suppl.3):55-8.

13. Cesari, M., Marco, P., Bartali, B., Antonio, Ch., Penninx, B., Williams, G., Atkinson, H., Antonio, M., Gurainik, J., Ferrucci, L. Los antioxidantes y el rendimiento físico en personas de edad avanzada: la Invecchiare in Chianti (In CHIANTI) un estudio. Am J Clin Nutr. 2004; 79 (2): 289-94.

14. González, G., García, D. Ejercicio físico y radicales libres, ¿es necesario una suplementación con antioxidantes?. Rev Internac Med Ciencias Actividad Física Deporte. 2012; 12 (46): 369-88.

15. Clarkson, P. Thompson, H., Antioxidants: what role do they play in physical activity and health?. Am J Clin Nutr. 2000; 72 (suppl.): $637 \mathrm{~s}-46 \mathrm{~s}$

16. Stanner, S., Hughes, J., Kelly, C., Buttriss, J. A review of the epidemiological evidence for the "antioxidant hypothesis". Public Health Nutr. 2004; 7 (3): 407-22.

17. Quiñones, M., Miguel, M., Aleixandre, A. Los polifenoles, compuestos de origen natural con efectos saludables sobre el sistema cardiovascular. Nutr Hosp. 2012; 27 (1): 76-89.

18. López, A., Fernando, C., Lazarova, Z., Bañuelos, R., Sánchez, S. Antioxidantes, un paradigma en el tratamiento de enfermedades. Rev Asociación Nac Científica Estudiantes Medicina (ANACEM). 2012; 6 (1): 48-53.

19. Li, Y., Ambrosone, Ch., Mc Cullough, M., Ahn, J., Stevens, V., Thun, M., Hong, Ch. Oxidative stress-related genotypes, fruit and vegetable consumption and breast cancer risk. Carcinogenesis. 2009; 30 (5): 777-84.

20. Delgado, L., Betanzos, G., Sumaya, M. Importancia de los antioxidantes dietarios en la disminución del estrés 
oxidativo. Investigación y Ciencia. 2010; (50), pp. 10-15.

21. Escamilla, Ch., Cuevas, E., Guevara, J. Flavonoides y sus acciones antioxidantes. Rev Fac Med. UNAM. 2009; 52 (2): $73-5$.

22. Muñoz, A., Ramos, D., Alvarado, C., Castañeda, B. Evaluación de la capacidad antioxidante y contenido de compuestos fenólicos en recursos vegetales promisorios. Rev Soc Quím Perú. 2007; 73 (3):142- 9.

23. Cunningham, D., Vannozi, S., Turk, R., Roderick, R., O'shea, E., Brilliant, K. Constituyentes fitoquímicos del arándano americano (Vaccinium macrocarpon) y sus beneficios para la salud. Rev Fitoterapia. 2005; 5 (1): 5-16.

24. Capocasa, F., Scalzo, J., Mezzetti, B., Battino, M. Combining quality and antioxidant attributes in the strawberry: The role of genotype. Food Chem, III. 2008; 872-8.

25. Ramírez, M., Geracitano, L., Marti, D., Henriques, A. Efectos beneficiosos de extractos de frutas rojas y de sus antocianos. Bol Latinoam Caribe Plantas Medicinales Aromáticas. 2009; 8 (6): 456-68.

26. Palomino, M., Guija, E., Lozano, N. Propiedades antioxidantes de la guayaba (Psidium guajava L.). Rev Soc Quím Perú. 2009; 75 (2): 227-34.

27. Marquina, V., Araujo, L., Ruiz, J., Rodríguez, A., Vit, P. Composición química y capacidad antioxidante en fruta, pulpa y mermelada de guayaba (Psidium guajava L.). Arch Latinoam Nutr. 2008; 58 (1): 98-102.

28. Perea, J., Cadena, T., Herrera, J. El cacao y sus productos como fuente de antioxidantes: Efecto del procesamiento. Salud Universidad Industrial Santander. 2009; 41: 128-34.

29. Sumaya, M., Suárez, T., Cruz, N., Alanís, E., Sanpedro, J. Innovación de productos de alto valor agregado a partir de la tuna mexicana. Rev Mexicana Agronegocios. 2010; XIV (27): 435-41.

30. López, L., García., S. Actividad antioxidante de extractos metanólicos y acuosos de distintas variedades de maíz mexicano. Nova Scientia. 2009; 2-1(3): 51-64.

31. Palomo, I., Fuentes, E., Carrasco, G., González, B., Moore, $R$. Actividad antioxidante, hipolipemiante y antiplaquetaria del tomate (Solanum lycopersicum L.) y el efecto de su procesamiento y almacenamiento. Rev Chil Nutr. 2010; 37 (4): 524-33.

32. Gutiérrez, A., Ledesma, L., García, I., Grajales, O. Capacidad antioxidante total en alimentos convencionales y regionales de Chiapas, México. Rev Cubana Salud Pública. 2007; 33 (1): 1-7.

33. Carvajal de Pabón, L., Turbay, S., Rojano, B., Álvarez, L., Restrepo, S., Álvarez, J., Bonilla, K., Ochoa, C., Sánchez, N. Algunas especies de Passiflora y su capacidad antioxidante. Rev Cubana Plantas Medic. 2011; 16 (4): 354-63.

34. Robles, M., Gorinstein, Sh., Martín, O., Astiazarán, H., González, G., Cruz, R. Frutos tropicales mínimamente procesados: potencial antioxidante y su impacto en la salud. Interciencia. 2007; 32 (4): 227-32. 